\title{
Approche fiabiliste du comportement des canalisations enterrées
}

\section{A. BENMANSOUR}

GEMCEA

149, rue Gabriel-Péri, 54000 Vandoeuvre-lès-Nancy et LG/ENSG

\section{G. AUVINET}

UNAM,

Ciudad Universitaria.

Apdo. Postal 70-472

Coyoacan, 04510, Mexico,

DF, Mexique

A.H. SOUBRA

ENSAIS

24, boulevard de la Victoire, 67084 Strasbourg
L'article s'intéresse au problème du comportement et de la sécurité des conduites enterrées. On présente d'abord les résultats de diverses études pathologiques qui ont permis de classer les principaux désordres rencontrés lors de l'inspection des réseaux d'assainissement. On évalue ensuite les apports des modélisations du système sol-conduite à une meilleure compréhension des mécanismes qui conduisent à ces désordres. Devant les incertitudes qui affectent les nombreux paramètres qui interviennent dans le comportement des conduites, une approche fiabiliste simplifiée est proposée pour

l'évaluation de leur sécurité. Ce type d'analyse permet de mettre en évidence les paramètres de dimensionnement qui contribuent à augmenter de manière significative la fiabilité de ces ouvrages.

\section{Reliability approach to buried pipes behavior}

The paper deals with the behavior and safety of buried pipes.
Results obtained from inspection of existing underground water and waste systems are reviewed. A classification of the main types of damage observed for these structures is established. An appraisal of the contribution of analytical and numerical modeling of the soil-pipe system to a better understanding of the failure mechanisms involved is presented. Taking into account that large uncertainties affect the parameters conditioning the behavior of these structures, a simplified reliability approach is proposed to evaluate their safety. This type of analysis is useful to identify design parameters that have the largest influence on the reliability of the system. 


\section{NOTATIONS}

AL : somme des charges des roues d'un convoi de type $\mathrm{B}_{\text {s }}$ sur un même axe.

B : largeur de la tranchée.

$\mathrm{COV}(, .$,$) : covariance entre deux variables aléatoires.$

D : diamètre extérieur de la conduite.

d : diamètre intérieur de la conduite.

e : épaisseur de la paroi de la conduite.

E. : module d'élasticité de la conduite.

$\mathrm{E}_{3} \quad$; module d'élasticité du sol environnant.

$E_{i+1} \quad$ : module d'élasticité du lit de pose.

$\mathrm{E}_{\text {eir }} \quad$; module d'élasticité de l'enrobage.

E $\quad$ : module d'élasticité đu remblaí.

$\mathrm{E}_{\mathrm{b}} \quad$ : module d'élasticité de la zone de bourrage.

$\mathrm{E}^{\prime} \quad$ : module de réaction du sol.

$G_{1} \quad$ : fonction de performance,

$\mathrm{H}$ : hauteur de recouvrement.

$\mathrm{K} \quad$ : coefficient de réaction du sol.

$\mathrm{K}^{\prime} \quad$ : rapport entre la poussée latérale et la pression verticale.

$\mathrm{K}_{0} \quad$ : facteur de charge dépendant de la hauteur de remblai.

L : longueur d'un élément de conduite.

LL : charge équivalente.

M : moment fléchissant dans la conduite.

$\mathrm{m}_{1} \quad$ : moyenne de la variable aléatoire $\mathrm{X}$.

$\mathrm{N} \quad$ : effort normal dans la conduite.

$\mathrm{OV}_{\text {dum }}$; ovalisation admissible de la conduite.

$\mathrm{P}$

p

p. : pression verticale au-dessus de la conduite.

$\mathrm{p}_{\mathrm{h}} \quad$ : pression latérale sur la conduite.

$\mathrm{X} \quad$ : variable aléatoire $\mathrm{i}$.

B : indice de fiabilité.

$\Delta \mathrm{x} \quad$ : déflexion horizontale de la conduite.

Ay $\quad$ : déflexion verticale de la conduite.

$\gamma \quad$ : poids spécifique du remblai.

$\mu \quad$ : tg $\phi$, coefficient de frottement interne du remblai.

$\mu^{\prime} \quad: \operatorname{tg} \phi^{\prime}$, coefficient de frottement sur la paroi de la tranchée.

$\sigma_{e} \quad$ : limite d'élasticité en compression du matériau de la conduite.

$\sigma^{\dagger} \quad$ : limite d'élasticité en traction du matériau de la conduite.

$\sigma \quad$ : écart type de la variable aléatoire $\mathrm{X}$. est difficile de modéliser les effets de façon réaliste. On connait par exemple l'importance des conditions de pose, des conditions géotechniques locales, des surcharges accidentelles. Ces éléments sont, dans une certaine mesure, aléatoires. Il est pourtant courant d'analyser les conduites au moyen de modèles déterministes basés sur des hypothèses simplificatrices (chargement symétrique, sol représenté de façon idéalisé) dont la valeur peut souvent être mise en doute. Il semble donc qu'il ait manqué aux multiples études théoriques et pratiques sur ce thème un cadre de référence général qui permette de confronter les modélisations et la réalité, et d'avancer effectivement dans la recherche d'une meilleure sécurité. Il est tentant pour définir ce cadre, d'avoir recours à l'approche fiabiliste qui s'avère utile pour déterminer les éléments (facteurs de robustesse) susceptibles de réduire, à moindre coût, les probabilités de désordres pour ce type d'ouvrage dans un contexte qui restera toujours incertain.

Nous présentons dans cet article une approche fiabiliste du comportement des conduites enterrées. Nous tentons en effet de faire le point sur la question en adoptant une vision globale et en recherchant le lien entre les désordres constatés sur les conduites d'assainissement et leur comportement mécanique. L'accent est donc mis sur les différentes études pathologiques et les enseignements qu'elles peuvent nous apporter. Bien qu'elles soient très utiles, ces études rencontrent rapidement leurs limites car elles nécessitent de nombreuses données dont on dispose rarement. Ce déficit est alors comblé par la modélisation du comportement du système sol-conduite. Sur la base des résultats des études pathologicques, la modélisation est orientée de sorte à couvrir la majorité des effets constatés. Ces effets sont étudiés en fonction de facteurs qui peuvent être accidentels, comme la présence de vides ou de points durs sous la conduite. Nous insistons sur leur prise en compte aussi bien en comportement transversal qu'en comportement axial de la conduite.

Nous introduisons par la suite, le concept de fiabilité et sa quantification au moyen des méthodes de premier ordre. Ces méthodes sont d'utilisation simple et permettent d'évaluer le (c poids $)$ de chaque paramètre du système sol-conduite sur la sécurité de ce dernier dans un contexte aléatoire. On s'intéresse dans cette approche à montrer l'effet des ( paramètres de contrôle ), paramètres de conception et de dimensionnement, sur la fiabilité de l'ouvrage dans des conditions de pose plus réalistes.

\section{Pathologie des canalisations enterrées}

\section{1}

\section{État actuel des réseaux d'assainissement en France}

Dans le domaine de la pathologie des conduites enterrées en général, et des conduites d'assainissement en particulier, le volume des travaux est assez restreint et présente un caractère disparate. Ceci témoigne, d'une part, d'une faiblesse du nombre de diagnostics réalisés actuellement et, d'autre part, de l'absence d'exploitation centralisée, rendant difficile toute analyse statistique. 
En France, les rares études consacrées au recensement des réseaux d'assainissement, permettent de tirer des informations sur les principales caractéristiques du patrimoine français. Ainsi, le linéaire national est estimé à environ $160000 \mathrm{~km}$, branchements particuliers non compris ; 60 à $75 \%$ des réseaux existants sont du type séparatif ; 70 à $75 \%$ des réseaux recensés sont non visitables. Les matériaux les plus utilisés pour la construction des réseaux d'assainissement sont le béton et l'amiante ciment qui représentent $70 \%$ du linéaire. Le grès est crédité de $10 \%$ environ, tandis que la fonte et le PVC ne totalisent que quelques pour cent. Plus de $30 \%$ du patrimoine a plus de 50 ans ; $8 \%$ du linéaire total, soit $8000 \mathrm{~km}$ de canalisations non visitables et $4300 \mathrm{~km}$ de canalisations visitables, nécessitent une réhabilitation (Schwarze, 1994; Degas, 1986). Ce dernier point montre l'ampleur des dégradations du patrimoine français. Les conséquences se font immédiatement sentir : de plus en plus, des symptômes de dégradation de la qualité des fleuves et des rivières qui traversent les grandes villes et qui constituent le principal milieu récepteur pour les rejets des zones urbaines, apparaissent. L'impact économique est aussi important: les collectivités en France consacrent chaque année 13 milliards de francs pour les systèmes d'assainissement. Il faut noter que la situation n'est guère meilleure dans les pays voisins (Aflak, 1994).

\section{Bilan des désordres les plus courants}

Un désordre est une particularité physique et/ou fonctionnelle de l'ouvrage, affectant ou susceptible d'affecter à terme la structure et/ou le fonctionnement du réseau. Les désordres sont de ce fait classés suivant deux catégories:

- les désordres de service ou de fonctionnement. On trouve dans cette catégorie les infiltrations et les exfiltrations d'effluents, les dépôts de matières solides, les contre-pentes, les déboitements et les décalages au niveau des joints :

- les désordres structuraux tels que les fissures, les cassures, les ovalisations et les effondrements.

Sur la fréquence de ces désordres, les résultats de différentes études pathologiques ne semblent pas toujours concorder. Cela n'est pas surprenant, puisque ces études se basent sur des données régionales, donc différentes d'une étude à une autre, et souvent incomplètes. Malgré les disparités qu'on a pu observer, il ressort que les défauts de type déboitement et décalage au niveau des joints sont les plus fréquents, suivis des fissures, longitudinales ou transversales, et des cassures. En effet, Lester et Farrar (1979) relèvent un taux de $58 \%$ de joints décalés ou déboîtés sur l'ensemble des désordres observés. De même, Le Barbanchan et Besanval (1986) constatent que le déboîtement et le décalage au niveau des joints constituent les défauts les plus courants avec un taux d'environ $40 \%$. Goulter et Kazemi (1989), analysant les dégradations du réseau de distribution d'eau potable de la ville de Winnipeg au Canada, remarquent, eux aussi, que les défauts des joints et les fissures sont parmi les désordres les plus courants avec des taux de $30 \%$ et $24 \%$ respectivement. Nous avons, de notre côté, pu confirmer ce résultat suite à une étude pathologique menée sur un linéaire d'environ $100 \mathrm{~km}$ du réseau d'assainissement de la communauté urbaine du Grand Nancy en France (Benmansour et Pilloy, 1996). En effet, les problèmes de déboîtement et de décalage au niveau des joints des canalisations représentent à eux seuls 30\% du pourcentage total des défauts. Les fissures représentent environ $20 \%$.

Lorsqu'ils ne peuvent être attribués à une mauvaise qualité de pose de la conduite, les problèmes de déboîtement et de décalage au niveau des joints sont souvent dus à un effet de flexion longitudinale de la canalisation, dont les causes sont multiples. Il en est de même pour la fissuration transversale. La fissuration longitudinale est, quant à elle, due à un effet d'écrasement ou de flexion transversale. Nous avons remarqué, en effet, que les fissures longitudinales étaient réparties aux quatre sommets de la conduite: clef de voùte, radier, reins droit et gauche. Nous avons constaté, par ailleurs, que le type de fissuration (longitudinale ou transversale) traduit un comportement bien particulier de la conduite, qui dépend des dimensions de celle-ci. A ce propos, la figure 1 montre, pour le cas de conduites en béton et béton armé, la relation entre le type de fissure et le paramètre $\mathrm{L} / \mathrm{D}$, L étant la longueur d'un élément de conduite et $\mathrm{D}$ son diamètre. Il est intéressant de remarquer que pour les fortes valeurs de $L / D$, on observe une tendance nette à la diminution du nombre de fissures longitudinales. Le comportement de la conduite est du type « poutre ». Inversement, le nombre de fissures transversales augmente avec ce rapport. Le comportement de la conduite dans ce cas est du type " anneau » (Benmansour et Pilloy, 1996 ; Benmansour et al., 1997).

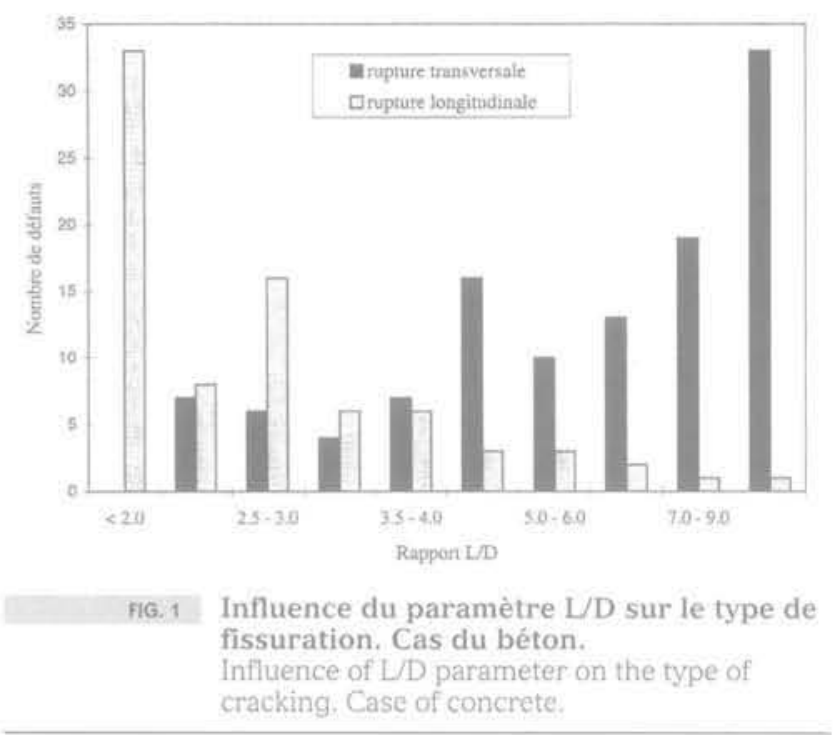

Les causes induisant des effets de flexion de la canalisation sont multiples. Les mouvements du sol encaissant sont les plus courants. En effet, les mécanismes géotechniques (tassements différentiels le long de la canalisation, discontinuités dans le sol, glissements de terrains...) combinés aux surcharges exceptionnelles de surface, sont à l'origine de bien des dégradations structurelles. La figure 2 montre à cet effet, l'influence de la profondeur de pose sur le taux des dégradations. Pour les conduites « superficielles $»$ (profondeur $<1.5 \mathrm{~m}$ ) le nombre de défauts est beaucoup plus élevé, probablement du fait des contraintes exceptionnelles en surface. Il est de l'ordre de quatre fois supérieur à la moyenne, toutes profondeurs confondues. 


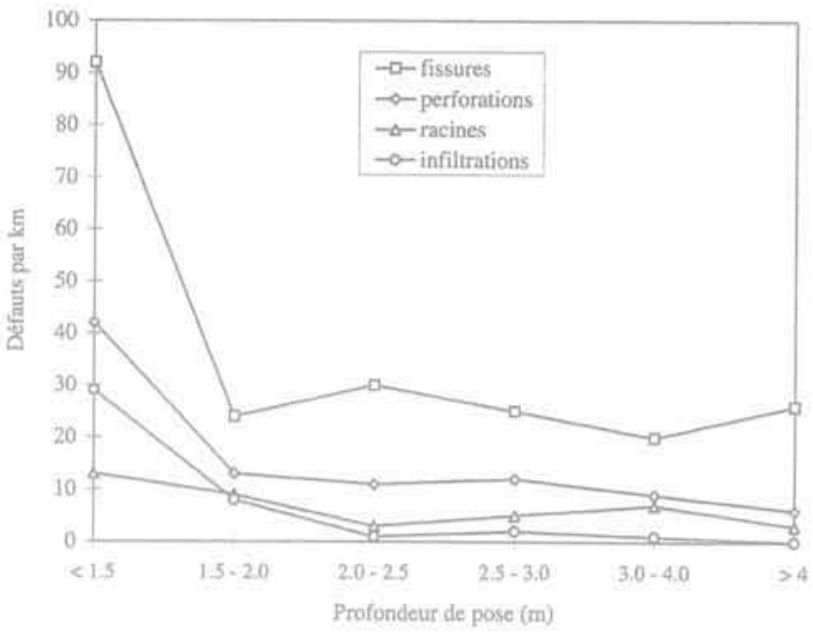

FIG.2 Influence de la profondeur de pose sur les désordres.

Influence of depth of trench on damage.

D'autres causes peuvent être citées, notamment la corrosion et les erreurs ou les insuffisances dans la conception. Ce dernier point mérite qu'on y prête attention. En effet, les règlements actuels, français, allemand ou américain, de vérification de la sécurité des conduites d'assainissement négligent le comportement axial et considèrent un comportement en déformation plane de ces ouvrages. Or, les études pathologiques citées plus haut montrent l'importance des désordres, en loccurrence les ruptures circulaires, traduisant ce genre de comportement (Benmansour et al., 1997),

Bien qu'elles soient très utiles, on se rend compte assez rapidement des limites des études pathologiques. Elles nécessitent en effet, de nombreuses données dont nous disposons rarement, et qui revêtent souvent un caractère fortement aléatoire. Ce déficit peut être alors comblé par la modélisation du comportement du système sol-conduite. Sur la base des résultats des études pathologiques, la modélisation peut être orientée de sorte à couvrir la majorité des effets constatés.

\section{3}

\section{Modélisation du comportement mécanique des conduites enterrées}

\section{1}

\section{Étude bibliographique des modèles de comportement des conduites enterrées}

La modélisation du comportement des conduites enterrées a été traitée dans la littérature suivant deux axes distincts : le comportement transversal et le comportement axial. Ce dernier n'a suscité que peu d'interêt. En effet, la plupart des auteurs considèrent un comportement de la conduite enterrée en déformation plane, ce qui n'est pas toujours réaliste.

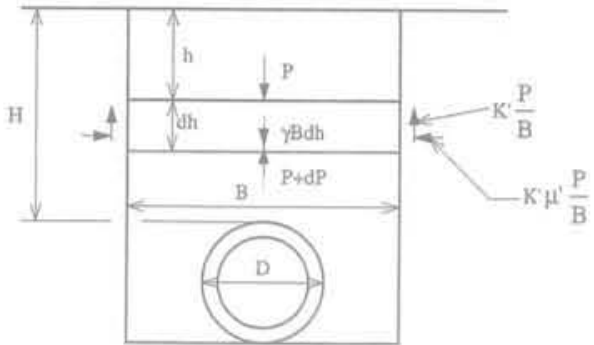

$$
\begin{aligned}
& \frac{\mathrm{dP}}{\mathrm{dh}}+\frac{2 K^{\prime} \mu^{\prime} \mathrm{P}}{\mathrm{B}}=\gamma \mathrm{B} \\
& \mathrm{P}=\frac{1-\mathrm{e}^{\left(-2 K \mu^{\prime} \cdot \frac{\mathrm{H}}{\mathrm{B}}\right)}}{2 \mathrm{~K}^{\prime} \mu^{\prime} \frac{\mathrm{H}}{\mathrm{B}}}
\end{aligned}
$$

FiG. 3 Principe de calcul du modèle de Marston. Principle of Marston's model.

\subsection{1 \\ Comportement transversal}

Pour ce qui concerne le comportement transversal, le modèle de Marston-Spangler est le plus ancien et constitue la base de la plupart des règlements actuels. En utilisant la théorie des silos et en considérant la tranchée comme un silo dont l'une des dimensions est infinie, Marston (1927) propose une méthode de calcul de la charge verticale supportée par la conduite. Cette charge correspond au poids des terres et, éventuellement, des surcharges de surface, diminué des forces de frottement le long des parois de la tranchée (Fig. 3). Cette charge est ensuite comparée à la résistance caractéristique de la conduite. Dans son approche, Marston ne s'intéresse qu'à la charge verticale concentrée au-dessus de la conduite. Ceci laisse supposer que le modèle n'est valable que dans le cas des conduites rigides. Par la suite, Spangler (1941) a généralísé le modèle de Marston aux conduites flexibles en considérant une distribution des contraintes autour de la conduite comme le montre la figure 4. Les pressions verticales sont distribuées le long du diamètre de la conduite et les pressions horizontales sur un arc de $100^{\circ}$. L'intensité de la pression horizontale est supposée proportionnelle à l'ovalisation horizontale. Le rapport des deux pressions verticale et horizontale dépend du degré de compacité du sol d'enrobage.

Par ailleurs, la méthode des éléments finis reste la plus efficace pour l'analyse de l'interaction sol-structure. Elle permet, notamment dans notre cas, de modéliser le contact entre la conduite et le sol (Valliappan et al., 1976), de prendre en compte le phasage de construction de la tranchée (Nath, 1977 ; Selig et al., 1982 ; Jeyapalan et Bolden, 1986 ; Selig et Packard, 1987) ou encore de considérer le comportement linéaire ou non-linéaire du sol et de la conduite (Nayak et al., 1977 ; Valliappan et al., 1976; Nath, 1977 ; Krizek et McQuade, 1978; Duncan, 1979; Selig et al., 1982). Ce dernier point est crucial. Il n'a cependant fait l'objet que d'un intérêt secondaire, L'utilisation de la méthode des éléments finis nécessite l'introduction de lois de comportement du sol et de la conduite. Si pour le matériau de la conduite, le comportement est relativement connu, il n'en est pas de même pour le matériau du remblai de la tranchée. En effet, sauf dans le cas d'études spécifiques où l'on dispose des résultats d'essais au laboratoire permettant de caractériser le comportement du matériau, il est difficile de choisir le modèle de comportement du sol de la tranchée. Même si un tel choix est fait, les paramètres du modèle sont souvent difficiles à déter- 


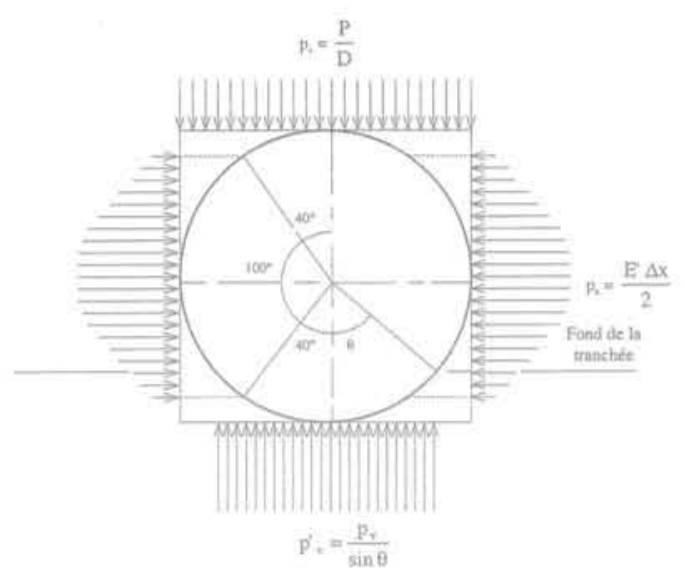

FIC. 4 Distribution des pressions autour d'une conduite flexible selon la méthode de Marston-Spangler.

Distribution of soil stresses around a flexible pipe according to the Marston-Spangler method.

miner. La loi hyperbolique de Duncan (Duncan et Chang, 1970) semble être la loi de comportement du remblai la plus utilisée dans l'analyse par éléments finis du système sol-conduite. Elle a fait l'objet de quelques comparaisons avec l'expérience et a donné des résultats satisfaisants (Duncan, 1979). De plus, dans une étude antérieure, nous avons pu confronter les résultats d'essais expérimentaux et ceux obtenus par une modélisation aux éléments finis en deux dimensions (Benmansour et al., 1995). Dans une tranchée expérimentale, où l'on a disposé des tuyaux de différentes natures (béton armé, fonte, amiante-ciment, PVC) et de différents diamètres (250,600 mm), on a mesuré les pressions autour de la conduite et les ovalisations de celle-ci. Nous avons alors comparé ces résultats à ceux donnés par la modélisation. Trois lois de comportement du matériau de remblai ont été considérées : loi élastique linéaire, loi élastique linéaire associée au critère de Mohr-Coulomb et loi de Cam-Clay. Nous avons trouvé que la loi élastique linéaire donnait des résultats très satisfaisants. Il semble donc que le comportement du remblai de la tranchée reste, dans bien des cas, dans le domaine élastique, ce que l'on peut attribuer à la forte préconsolidation des sols compactés. Ce comportement est plus sensible aux variations des propriétés du sol, et par conséquent aux variations des paramètres de la loi de comportement, qu'à la forme de celle-ci.

Notons enfin que l'une des difficultés majeures du modèle en comportement transversal est la prise compte des surcharges superficielles. En effet, il est difficile, dans l'hypothèse de la déformation plane, de prendre en compte les surcharges superficielles et, notamment, celles dues aux véhicules. Nous avons choisi dans cette étude de remplacer la charge d'un véhicule par une charge équivalente en deux dimensions, comme cela a été proposé par de nombreux auteurs. Duncan et Drawsky (1983), se basant sur la théorie de Boussinesq, suggèrent que la charge équivalente en déformation plane est celle qui produirait la même contrainte verticale sur la conduite que celle produite par un chargement de véhicule en trois dimensions. Ils proposent la formule suivante :

$$
\mathrm{LL}=\frac{\mathrm{AL}}{\mathrm{K}_{0}}
$$

\section{LL : charge équivalente ;}

$\mathrm{AL}$ : somme des charges des roues sur un même axe : $\mathrm{K}_{0}$ : facteur de charge dépendant de la hauteur de remblai.

Seed et Raines (1988) font remarquer que cette formule est conservatrice. De nouvelles méthodes, plus rigoureuses, basées sur un couplage entre la méthode des éléments finis et les transformées de Fourier ont été proposées récemment (Fernando et al., 1996). Nous avons choisi d'utiliser la méthode de Duncan et Drawsky (1983) pour sa facilité de mise en œuvre.

\subsection{2 \\ Comportement axial}

Pour le comportement axial de la conduite, le modèle le plus utilisé dans le domaine des fondations, est celui de la poutre sur appui élastique continu. $\mathrm{Ce}$ modèle considère que la poutre (conduite), dans le sens longitudinal, est continue et élastique. Le sol supportant l'ouvrage est aussi considéré élastique linéaire. Sous le chargement, la poutre (conduite) fléchit et se déforme suivant une courbe en cloche. On suppose alors que la réaction du sol, ou pression de contact, est proportionnelle à la flèche de la conduite suivant la formule de Terzaghi (Fig. 5). En appliquant les équations de base de la résistance des matériaux, on obtient l'équation différentielle du 4 e ordre de la ligne élastique de la conduite, dont la résolution donne l'effort tranchant et le moment fléchissant en chaque section de la structure.

Le comportement axial des conduites enterrées n'a fait l'objet que de peu d'études. On a souvent considéré des conditions de pose optimales assurant un comportement en déformation plane de la conduite. Or l'expérience montre que ces conditions optimales sont loin d'être toujours respectées dans la réalité: En effet, suite à des mécanismes géotechniques (tassements différentiels, dégradations du lit de pose...), la conduite, d'une structure initialement sur appui continu, devient une structure sur appuis espacés. Afin de tenir compte de ces éventuelles discontinuités (vides sous la conduite. tassements locaux, joints de la conduite...), certains auteurs ont introduit les méthodes numériques (éléments finis, couplage éléments finis-équations intégrales) pour étudier le comportement de l'ouvrage dans ces conditions (Selvadurai, 1985, 1991 ; Selvadurai et Pang, 1988 ; Selvadurai et al., 1990 ; Jeyapalan et Abdel-Magid, 1987). Ces études ont surtout montré l'importance de la prise en compte du comportement axial de la conduite dans des conditions de service plus réalistes.

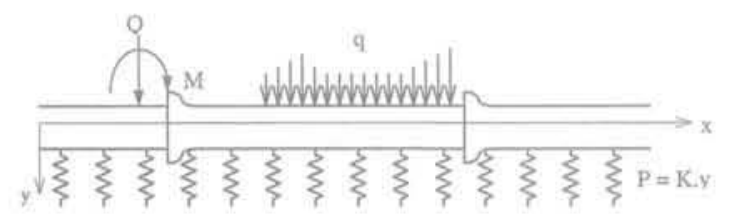

FG.5 Modèle de la poutre sur appui élastique continu.

Model of the beam on continuous elastic support. 


\section{Modélisation du comportement transversal et axial de la conduite}

Suite aux constatations pathologiques, nous nous sommes intéressés aux causes éventuelles qui peuvent conduire à un comportement limite en flexion transversale ou longitudinale de la conduite. Ce sont essentiellement les conditions de pose de la canalisation, les surcharges excessives de surface, les discontinuités dans le sol, les vides et les points durs sous la conduite, etc.

Dans un souci de simplification, nous avons divisé le problème en considérant, d'une part, le comportement transversal et, d'autre part, le comportement axial. Le comportement transversal de la conduite a été modélisé à l'aide du logiciel CESAR, du Laboratoire central des ponts et chaussées (LCPC), qui est un progiciel général de calcul par la méthode des éléments finis adapté aux problèmes de génie civil. Le comportement axial a été modélisé à l'aide du logiciel RIDO (Fages et Bouyat, 1971) basé sur la méthode du coefficient de réaction. Il est particulièrement adapté aux structures linéaires, telles que les pieux ou les conduites. La figure 6 présente un exemple de maillage des deux modèles utilisés.

Nous nous limitons dans ce qui suit à la présentation des résultats de deux cas montrant l'influence :

- des conditions de pose sur le comportement transversal de la conduite ;

- de la présence d'une discontinuité dans sol le long de la canalisation sur le comportement axial.

\section{- Cas 1}

Le premier cas correspond à une conduite en béton armé de 2 m de diamètre (D2000 BA) dans une tranchée de 2,5 $\mathrm{m}$ de largeur et avec une hauteur de recouvrement de $4 \mathrm{~m}$. La conduite est soumise, en plus des charges des terres, aux surcharges de véhicule de type convoi B (chargement considéré dans le règlement français et correspondant au croisement de deux camions de 30 tonnes). On admet que la conduite se trouve dans des conditions de pose médiocres et que la compacité du lit de pose à proximité des hanches de la conduite est faible (module de la zone de bourrage $E_{b}$ ), suite, par exemple, à une mauvaise mise en place ou à un entrainement hydrodynamique des fines. La figure 7 montre la distribution du moment fléchissant autour de la conduite. On considère une adhérence parfaite entre le sol et la conduite. Le calcul est fait en élasticité linéaire.

Nous constatons que le moment fléchissant au niveau du radier de la canalisation est très sensible à la compacité de la zone de bourrage. Il augmente d'environ $85 \%$ par rapport au cas homogène (conditions de pose optimales). La diminution de la compacité d'une partie du lit de pose provoque une amplification de l'effet d'écrasement de la conduite. Ceci fait augmenter les efforts dans la conduite, notamment au niveau du radier. Cela montre l'importance de la zone de bourrage et le rôle qu'elle peut jouer dans la dégradation de la conduite.

\section{- Cas 2}

Dans le second cas nous considérons un tronçon de canalisation entre deux regards de visite long de $30 \mathrm{~m}$
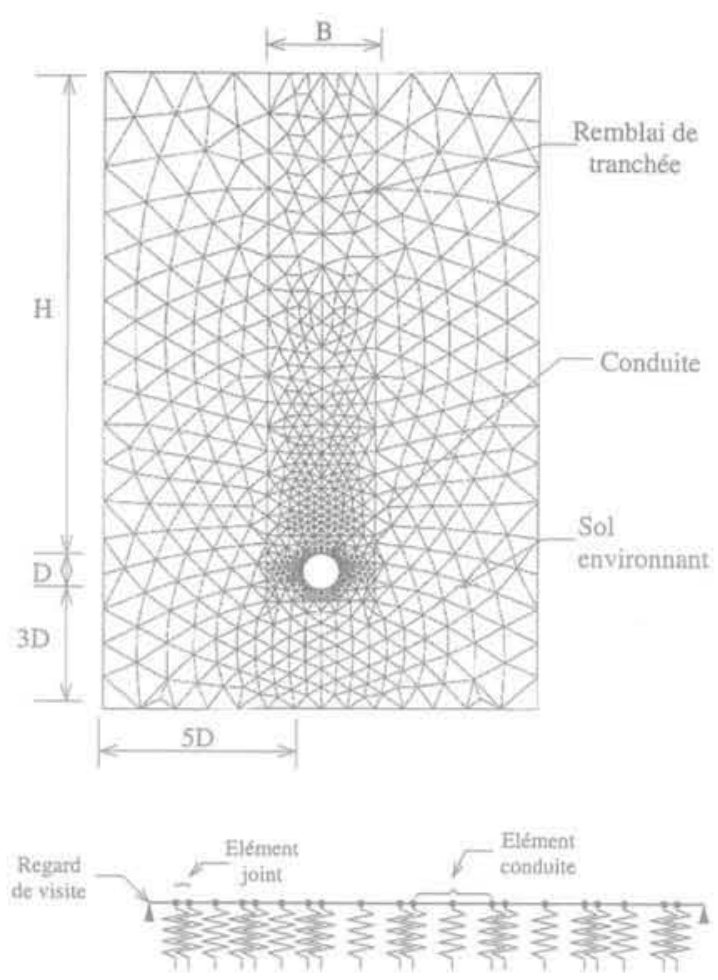

(b)

FIG.6 Maillage du système sol-conduite. Mesh of the soil-pipe system.

constitué d'éléments de conduite en béton armé de $300 \mathrm{~mm}$ de diamètre et de $3 \mathrm{~m}$ de longueur. Les regards de visite sont modélisés par des appuis simples qui traduisent la souplesse de la canalisation à ces endroits. Le tronçon traverse un sol dont les caractéristiques de déformation (module du sol) changent brutalement. La figure 8 montre la variation du moment fléchissant maximal, rapporté à celui d'un sol sans discontinuité $\left(\mathrm{M}_{\text {max }}\right.$ : moment maximal dans le cas du sol non homogène ; $M_{\text {hmax }}$ : moment maximal dans le cas d'un sol homogène), en fonction du rapport des modules des deux sols rencontrés.

Le moment maximal se trouve à l'interface des deux sols. Nous remarquons qu'une discontinuité dans le sol le long de la conduite provoque une augmentation considérable du moment, notamment pour les faibles valeurs du rapport $E_{\mathrm{s}} / \mathrm{E}_{\mathrm{s} 1}$. Ainsi pour des sols très différents $\left(E_{\mathrm{s} 2} / E_{s 1}=0.1\right)$, le moment maximal est de 14 (tuyaux sans joints) à 18 fois (tuyaux avec joints) supérieur à celui du cas d'un sol homogène $\left(\mathrm{E}_{\mathrm{e}} / \mathrm{E}_{\mathrm{y}}=1\right)$. Nous constatons par ailleurs que pour $\mathrm{E}_{\mathrm{s}} / \mathrm{E}_{\mathrm{s1}}=0,5 \mathrm{la}$ discontinuité dans le sol se fait réellement sentir.

Hormis ces deux applications, nous avons étudié l'effet d'un ensemble de facteurs d'environnement et de dimensionnement du système sol-conduite sur le comportement de cette dernière. Cette étude paramétrique nous a permis, d'une part, de confirmer les résultats de l'étude pathologique (comportement en flexion transversale et longitudinale, influence de la profondeur de pose, influence de l'élancement L/D) et, d'autre part, de justifier l'utilisation des modèles présentés dans l'analyse de fiabilitê (Benmansour, 1996). 


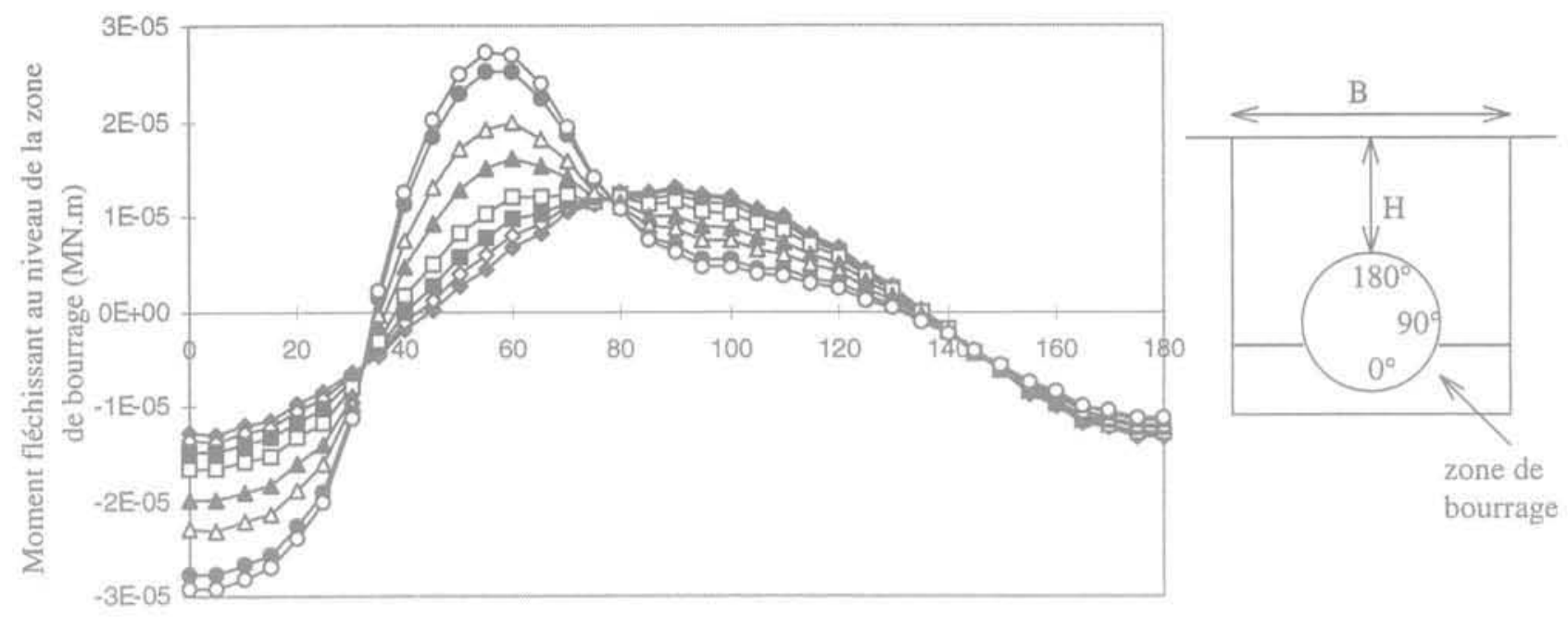

Angle de circonférence $\left({ }^{\circ}\right)$

$E_{\mathrm{b}}(\mathrm{MPa})$

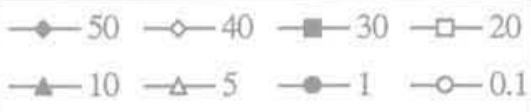

$\mathrm{E}_{\mathrm{c}}=40000 \mathrm{MPa}$

FG. 7 Moment fléchissant autour de la conduite. Cas d'un D2000 BA.

Bending moment diagram around the pipe, Case of D2000 BA.

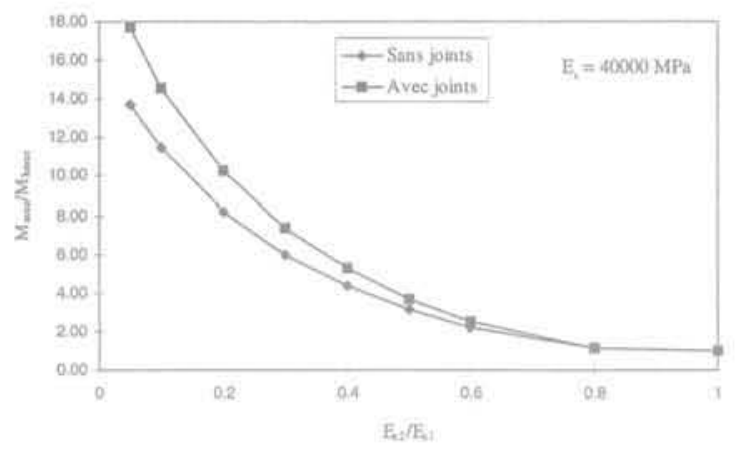

FIG. 8 Variation du moment fléchissant maximal relatif en fonction du rapport $\mathrm{E}_{\mathrm{s} 2} / \mathrm{E}_{\mathrm{s} 1}$. Variation of maximal bending moment with ratio $E_{2,} / E_{s i}$

\section{4}

\section{Analyse de fiabilité}

\section{Introduction}

Les nombreuses variables qui affectent le comportement des conduites enterrées sont le plus souvent difficiles à évaluer au niveau du projet. Ceci est particulièrement vrai pour les paramètres géotechniques des matériaux en place, dont les variations naturelles sont souvent imprévisibles, et des matériaux de remblai. sensibles aux conditions de mise en place. Il est donc attrayant d'appliquer à ce problème les techniques de l'analyse de fiabilité en considérant ces quantités comme des variables aléatoires. Une analyse de ce type permet, en effet, d'évaluer la sécurité de l'ouvrage en tenant compte du poids respectif de l'incertitude qui affecte chacune des variables. Elle permet surtout de comparer les probabilités de ruine associées à diverses options et de définir les paramètres de dimensionnement sur lesquels il est possible de jouer pour arriver à des solutions robustes, peu sensibles aux incertitudes.

Les conduites enterrées sont des systèmes complexes, dont les caractéristiques évoluent avec le temps. Une analyse rigoureuse de fiabilité pose donc des problèmes délicats, pour l'instant sans doute insurmontables. On peut, toutefois, dans un premier temps limiter l'analyse à certains composants du système ; une section transversale typique de la conduite pour le comportement transversal et un tronçon typique pour le comportement axial. Cette analyse peut, par ailleurs, se faire dans un cadre similaire à celui des analyses déterministes habituelles. Le temps n'intervient alors qu'au travers du concept de vie utile de l'ouvrage. Les sollicitations externes considérées sont les plus défavorables prévues durant cette période et on admet que les propriétés mécaniques considérées (et l'incertitude qui les affecte) restent constantes durant cette même période.

\section{2}

\section{Principe du calcul de fiabilité}

Le principe de calcul de fiabilité, tel qu'il a été utilisé dans cette étude, est schématisé sur la figure 9,

Le système sol-conduite est défini par ses variables déterministes et aléatoires. Les deux modèles de comportement servent à déterminer les valeurs des efforts internes dans chaque section de la conduite, en fonction des valeurs prises par les variables aléatoires de base. Ces efforts sont, pour le comportement transver- 


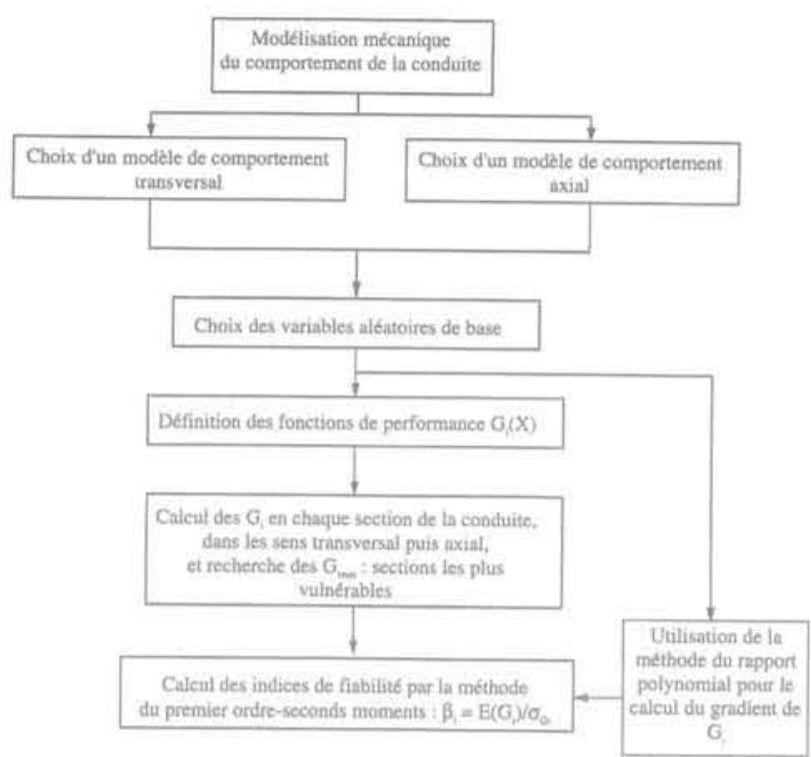

Principe du calcul de fiabilité.

Principle of reliability calculation.

sal, le moment fléchissant $M$, l'effort normal $N$ et l'effort tranchant T. Pour le comportement axial, ce sont, le moment fléchissant $M$ et l'effort tranchant $T$. Les fonctions de performance $G_{\text {i sont alors exprimées }}$ en terme de ces efforts. Nous recherchons la section la plus vulnérable qui donne la valeur de $G$ minimale. Cette section correspond à l'indice de fiabilité $\beta$ le plus faible. On procède alors au calcul de l'indice de fiabilité $\beta_{\text {i }}$ par la méthode du premier ordre-seconds moments.

\section{3}

\section{Définition du système et de ses variables}

Le système sol-conduite est schématisé sur la figure 10 :

Le système représente un tronçon de canalisation d'environ $30 \mathrm{~m}$ de longueur entre deux regards de visite. Les deux éléments du système sont le sol et la conduite. Le sol est composé du sol existant ou environnant et du sol de la tranchée, constitué du lit de pose, de l'enrobage et du remblai. Chacun de ces sols est caractérisé par ses propriétés physiques et mécaniques. La conduite est caractérisée par ses dimensions, sa nature et ses propriétés mécaniques et physiques. L'ensemble du système peut être soumis à des sollicitations externes telles que les surcharges de surface statiques ou dynamiques.

Les variables du système sont de deux types : les variables déterministes, ce sont toutes les dimensions du système (hauteur de recouvrement, largeur de tranchée, diamètre de la conduite, longueur de la conduite...) et les variables aléatoires, ce sont les propriétés des sols et de la conduite, notamment les modules des sols et les résistances à la traction et à la compression du matériau de la conduite.

Il est nécessaire d'associer aux variables aléatoires une moyenne et un coefficient de variation. Dans notre approche nous considérons que les moyennes des variables aléatoires reflètent le niveau de qualité attendu, et les coefficients de variation cherchent à couvrir les différences possibles entre les valeurs des paramètres estimées ou souhaitées et les valeurs réelles, du fait de plusieurs facteurs comme l'hétérogénéité du sol naturel, des matériaux de construction disponibles, de la qualification de l'entreprise, du contrôle de qualité mis en place, des conditions climatiques durant la construction, etc. Les valeurs retenues seront finalement le reflet, souvent subjectif, de l'expérience de celui ou de ceux qui réalisent l'analyse de fiabilité dans un contexte donné. On pourrait souligner, à juste titre, que les résultats d'une analyse de fiabilité peuvent ainsi varier d'un analyste à un autre. Cela est vrai, mais, à mème niveau de compétence technique, on peut s'attendre à ce que les grandes tendances des résultats de l'analyse restent les mêmes.

Les valeurs retenues pour les coefficients de variation sont indiquées dans le tableau suivant:

avec

$\mathrm{E}_{\mathrm{s}}$ : module du sol environnant ;

$\mathrm{E}_{\text {lit }}$ : module du lit de pose;

$\mathrm{E}_{\text {enr }}$ : module de l'enrobage;

$E_{\mathrm{r}}$ : module du remblai ;

$\sigma_{e^{c}}^{c}, \sigma_{e}^{t}$ : limites d'élasticité en compression et en traction respectivement, du matériau de la conduite :

$\mathrm{OV}_{\mathrm{adm}}$ : ovalisation admissible da la conduite;

$\mathrm{K}$ : coefficient de réaction du sol.

\section{4}

\section{Définition des fonctions de performance}

La fonction de performance G, ou fonction d'état limite, est une fonction des variables aléatoires $\mathrm{X}$ qui permet de séparer la zone de sécurité du système de sa zone de ruine, de telle sorte que :

$G(X)>0$ correspond à l'état de sécurité du système ; $\mathrm{G}(\mathrm{X})=0$ correspond à l'état limite du système :

$G(X)<0$ correspond à l'état de ruine du système.

Pour un système sol-conduite présentant plusieurs types de désordres, ou d'états limites, nous pouvons définir plusieurs fonctions de performance, Cependant, nous avons constaté lors de l'étude pathologique que les effets (fissures longitudinales, fissures circulaires, déboîtements) dus aux comportements en flexion transversale et en flexion longitudinale de la conduite étaient les plus fréquents (Benmansour, 1996). C'est par rapport à ces effets que nous avons défini nos fonctions de performance.

TABLEAU Coefficients de variation des variables aléatoires du système.

Coefficients of variation of randon variables of the system.

\begin{tabular}{l|l}
\hline \multicolumn{1}{c|}{ Variables aléatoires } & $C V$ \\
\hline$E_{r^{\prime}}, E_{u r}, E_{e n r} E_{r}$ & 0,4 \\
\hline$\sigma_{e}^{c}, \sigma_{e}^{c}$ & 0,25 \\
\hline$K$ & 0,4 \\
\hline
\end{tabular}




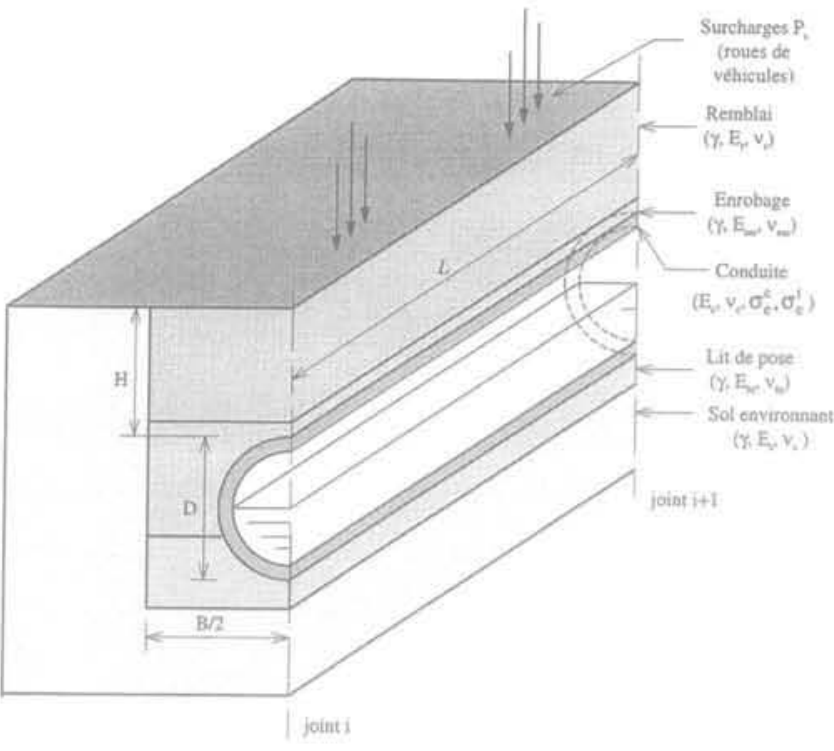

FiG. 10 Système sol-conduite. Soil-pipe system.
En comportement transversal, nous avons considéré un comportement élastique parfaitement plastique du matériau de la paroi de la conduite soumise au moment fléchissant et à l'effort normal (Fig. 11.a). Les équations d'équilibre des forces de la paroi de la conduite totalement plastifiée conduit à la première fonction de performance $\mathrm{G}_{1}$ vis-à-vis de la flexion composée en comportement transversal :

$$
G_{1}=\left|\frac{2 M}{M_{p l}^{t}+M_{p l}^{c}}\right|+\left(\frac{N}{N_{m}}\right)^{2}-2 \frac{|N|}{N_{m}} \frac{\sigma_{e}^{c}-\sigma_{e}^{t}}{\sigma_{e}^{c}+\sigma_{e}^{t}}+\frac{\left(\sigma_{e}^{c}-\sigma_{e}^{t}\right)^{2}}{\left(\sigma_{e}^{c}-\sigma_{e}^{t}\right)^{2}}-1
$$

avec

$$
\begin{aligned}
& M_{p !}^{c}=\frac{e^{2}}{4} \sigma_{e}^{c} ; \\
& M_{p t}^{i}=\frac{e^{2}}{4} \sigma_{e}^{t} ; \\
& N_{m}=\frac{\sigma_{e}^{c}+\sigma_{e}^{t}}{2} e ;
\end{aligned}
$$

$\sigma_{e}^{\leftarrow}$ : limite d'élasticité en compression :

$\sigma_{e}^{t}$ : limite d'élasticité en traction;

e : épaisseur de la paroi de la conduite.

De même, les équations d'équilibre de la section plastifiée de la conduite soumise au moment fléchissant conduit à la deuxième fonction de performance $\mathrm{G}_{2}$ visà-vis de la flexion simple en comportement axial (Fig. 11 b) :

$$
G_{2}=\frac{\left(D^{3}-d^{3}\right)\left(\sigma_{e}^{e}-\sigma_{e}^{t}\right)}{12|M|}-1
$$

\section{Calcul de fiabilité}

Comme nous avons pu le constater, les fonctions de performances sont non-linéaires et sont fonctions implicites des variables aléatoires. Dans ces conditions, il est difficile d'estimer la probabilité de ruine du sys-
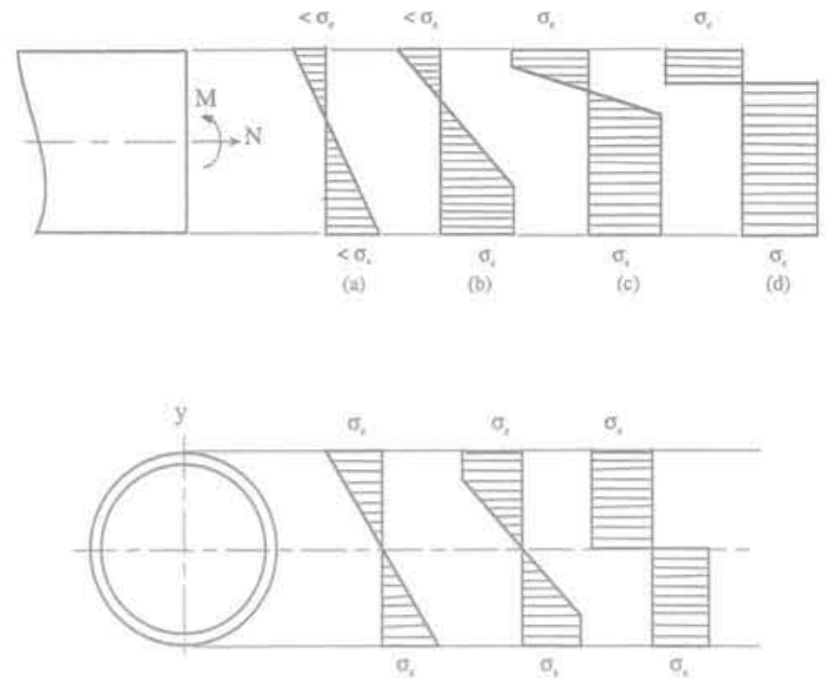

(b)

(c)

FG. 11 Plastification (a) de la paroi et (b) de la section de la conduite.

Yield of (a) the wall and (b) the section of the pipe.

tème. On introduit à sa place l'indice de fiabilité dont la définition la plus simple est donnée par Cornell: Cette définition est utilisée dans cette approche et est définie comme suit :

$$
\beta=\frac{E[G(X)]}{\sigma_{G(X)}}
$$

$\beta$ : indice de fiabilité ;

$\mathrm{E}[\mathrm{G}(\mathrm{X})]$ : espérance mathématique de $\mathrm{G}(\mathrm{X})$; $\sigma_{G(X)}:$ écart type de $G(X)$.

L'estimation de cet indice s'effectue à l'aide d'un programme de calcul basé sur la méthode du premier ordreseconds moments, plus connue sous le nom de la méthode des premiers moments. Dans cette méthode; si la fonction $\mathrm{G}$ est différentiable par rapport aux variables aléatoires $\mathrm{X}$, analytiquement ou numériquement, elle peut être developpée en série de Taylor autour de la valeur moyenne du vecteur X. En limitant le développement au premier ordre, on obtient une expression approchée de $\mathrm{G}$, qui permet de déterminer plus simplement les valeurs de l'espérance mathématique et de l'écart type de G (Benjamin et Cornell, 1970). Ainsi, on a :

$$
\begin{gathered}
E[G(X)]=G\left(m_{1}, m_{2}, \ldots, m_{n}\right) \\
{\left[\sigma_{G(X)}\right]^{2}=\left.\left.\sum_{i=1}^{n} \sum_{j=1}^{n} \frac{\partial G(X)}{\partial X_{i}}\right|_{\left(m_{1}, \ldots, m_{n}\right.} \frac{\partial G(X)}{\partial X}\right|_{\left(m_{1}, \ldots, m_{n}\right)} \operatorname{COV}\left(X_{1}, X_{j}\right)}
\end{gathered}
$$

$\mathrm{m}_{1}$ : moyenne de la variable aléatoire $\mathrm{X}$;

$\operatorname{COV}(\mathrm{X}, \mathrm{X})$ : covariance des deux variables aléatoires $\mathrm{X}$ et $\mathrm{X}$.

$G_{1}$ et $G_{2}$ étant des fonctions implicites des variables aléatoires de base, le calcul du gradient de $G_{1}$ par rapport aux variables aléatoires $X$ se fait par la méthode numérique du rapport polynomial.

Nous nous intéressons dans cette approche à la variation des indices de fiabilité $\beta_{1}$ et $\beta_{2}$, relatifs à $G_{1}$ et $G_{3}$, en fonction des différents paramètres du système sol-conduite que nous avons classé dans trois catégories (tableau II) : 
TABLEAU II Les trois classes de paramètres du système sol-conduite. The three types of parameters of the soil-pipe system.

\begin{tabular}{l|l|l}
\multicolumn{1}{c|}{ Paramètres de contrôle } & \multicolumn{1}{c|}{ Paramètres intermédiaires } & Paramètres accidentels \\
\hline Profondeur de pose & $\begin{array}{l}\text { Bourrage au niveau des hanches } \\
\text { de la conduite }\end{array}$ & Vides sous la conduite \\
\hline Largeur de la tranchée & $\begin{array}{l}\text { Caractéristiques mécaniques } \\
\text { du matériau de remblaí }\end{array}$ & Points durs sous la conduite \\
\hline Épaisseur de la paroi de la conduite & $\begin{array}{l}\text { Caractéristiques mécaniques } \\
\text { du matériau de la conduite }\end{array}$ & $\begin{array}{l}\text { Discontinuité du sol le long } \\
\text { de la concluite }\end{array}$ \\
\hline Élancement L/D & Asymétrie géométriqué & Surcharges excessives \\
\hline Flexibilíté des joints & &
\end{tabular}

- les paramètres de contrôle sont des paramètres déterministes dont les valeurs et les variations peuvent être facilement contrôlées, telles que les dimensions de la conduite ou de la tranchée. Ceux parmi ces paramètres, dont l'influence sur les indices de fiabilité est grande sont les facteurs de robustesse du système :

- les paramètres accidentels sont des paramètres aléatoires, sur lesquels nous n'avons aucune emprise, tels que les vides sous la conduite, les points durs ou les discontinuités dans le sol:

- les paramètres intermédiaires peuvent constituer des paramètres de contrôle en prenant les mesures nécessaires pouren assurer le suivi, tels que la qualité de bourrage au niveau des hanches de la conduite ou celle du compactage du remblai de la tranchée. Dans le cas contraire, ils deviennent des paramètres accidentels.

L'intérêt d'une telle classification est de constater l'influence des paramètres de contrôle sur la fiabilité du système dans des conditions ( normales ) de pose et de conception, et d'évaluer leur effet sur la fiabilité du système en présence d'événements accidentels. Afin d'illustrer cette démarche, nous présentons dans ce qui suit deux exemples de variation de l'indice de fiabilité en fonction de certains paramètres de contrôle.

\section{6}

\section{Résultats numériques}

\section{9 \\ Influence de la profondeur de pose}

La figure 12 montre la variation de l'indice de fiabilité $\beta$ en fonction de la profondeur de pose $\mathrm{H}$ pour les cas d'un D300 BA et d'un D800 BA en présence de surcharges de surface.

Nous remarquons que les valeurs de $\beta$, sont plus faibles dans le cas du D800. En effet, l'étude paramétrique a montré que les efforts sont d'autant plus importants que la rigidité de la paroi de la conduite est plus grande. Par ailleurs, l'indice $\beta$, augmente avec la profondeur de pose. Cette augmentation est d'environ $30 \%$ pour le D800, lorsque la hauteur de recouvrement passe de $0,8 \mathrm{~m}$ à $6 \mathrm{~m}$. Ceci peut s'expliquer par le fait qu'en présence de surcharges superficielles les efforts diminuent avec la profondeur de pose faisant augmenter la fiabilité de la conduite.

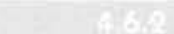

\section{Influence du paramètre d'élancement L/D}

La figure 13 montre la variation de l'indice de fiabilité $\beta$, en fonction de l'élancement de la conduite L/D dans les cas d'un « bon sol », ou sol non-compressible, et celui d'un " mauvais sol ), ou sol très compressible.

Nous remarquons une nette différence entre les valeurs de l'indice $\beta_{2}$ correspondant à un « bon sol $)$ et celles correspondant à un ( sol médiocre ») ces dernières étant plus faibles. En effet, l'étude paramétrique a montré que les efforts internes augmentent considérablement lorsque le module du sol, ou le coefficient de réaction, diminue, entraînant une diminution de la fiabilité de l'ouvrage (Benmansour, 1996).

Nous constatons par ailleurs que la variation de l'indice $\beta_{2}$ avec $L / D$ confirme les résultats des études pathologique et paramétrique. Plus le rapport L/D est élevé, plus fort est le moment fléchissant dans la conduite, plus grande est la probabilité d'apparition de fissures circulaires, et plus faible est donc l'indice de fiabilité. Cependant, comme on pouvait s'y attendre, cette tendance de l'indice $\beta_{2}$ avec L/D est plus marquée dans le cas du " sol médiocre ». Ainsi, dans le cas d'un "sol médiocre », $\beta$, diminue d'environ $15 \%$ lorsque $\mathrm{L} / \mathrm{D}$ passe de 1 à 10 , tandis que dans le cas d'un "bon

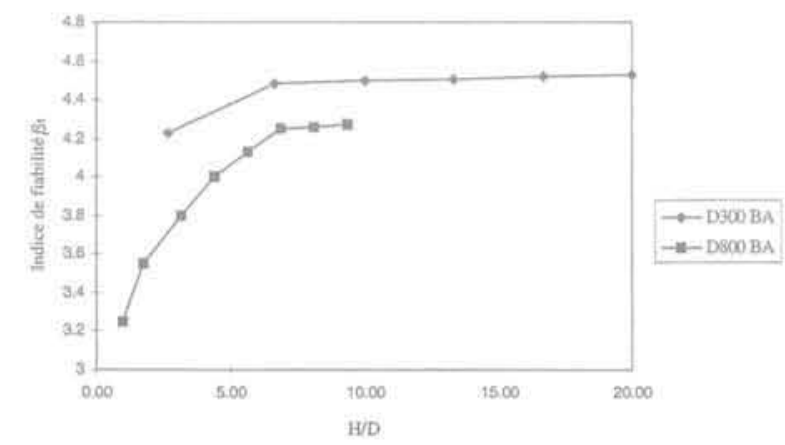

FG. 12 Variation de l'indice de fiabilité $\beta$, en fonction de la profondeur de pose. Variation of reliability index $\beta$, with the trench's depth. 


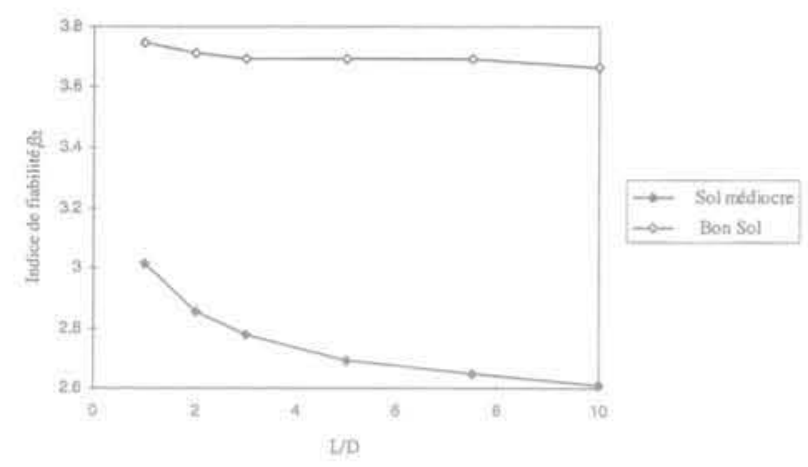

FIG.13 Variation de l'indice de fiabilité $\beta_{2}$ en fonction du paramètre $L D$. Variation of reliability index $\beta$, with the parameter $\mathrm{L} / \mathrm{D}$.

TABLEAU III Effet de la profondeur de pose. Effect of depth.

\begin{tabular}{l|c}
\hline \multicolumn{1}{c|}{ Conditions de pose } & $\beta_{3}$ \\
\hline bon bourrage, $H+D=2,8 \mathrm{~m}$ & 3,98 \\
\hline mauvais bourrage, $\mathrm{H}+\mathrm{D}=2,8 \mathrm{~m}$ & 0 \\
\hline mauvais bourrage, $\mathrm{H}+\mathrm{D}=3,8 \mathrm{~m}$ & 1,84 \\
\hline
\end{tabular}

TABLEAUIV Effet du paramètre L/D. Effect of parameter L/D.

\begin{tabular}{|c|c}
\hline \multicolumn{1}{|c|}{ Caractéristiques de la conciuite } & $\beta_{2}$ \\
\hline $\mathrm{L}=3 \mathrm{~m}, \mathrm{~L} D=3$ & 2,52 \\
\hline $\mathrm{L}=1 \mathrm{~m}, \mathrm{~L} / \mathrm{D}=1$ & 3,37 \\
\hline
\end{tabular}

sol $x$ la diminution n'est que de $4 \%$. Le paramètre L/D joue donc un rôle, sur la fiabilité de la conduite, d'autant plus important que cette dernière se trouve dans un sol médiocre.

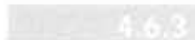 \\ Influence des paramètres de contrôle en présence d'événements accidentels}

Pour les deux exemples précédents, nous avons constaté l'influence notable des paramètres de contrôle sur les indices de fiabilité. Nous pouvons mieux apprécier l'influence de ces paramètres en introduisant un événement accidentel. Prenons l'exemple d'une conduite en béton armé de diamètre $2 \mathrm{~m}$ (D2000 BA) sous une hauteur de couverture de $0.8 \mathrm{~m}$ et une surcharge de type convoi B. Dans les conditions d'une mauvaise qualité de bourrage, nous augmentons la profondeur de pose de $1 \mathrm{~m}$, nous obtenons les résultats du tableau III. L'autre exemple concerne un D1000 BA soumis aux mêmes charges que précédemment et passant d'une zone stable à une zone instable. Nous faisons varier la longueur de l'élément de la conduite, nous obtenons alors les résultats du tableau IV. Nous constatons que, même en présence d'un événement accidentel, les paramètres de contrôle permettent d'améliorer la fiabilité de la conduite. D'autre part, ces résultats nous permettent d'estimer le « risque » pris en pré- sence d'un événement accidentel lorsque les paramètres du projet sont fixés.

Nous pouvons appliquer ce type de raisonnement en représentant la variation de l'indice de fiabilité en fonction de plusieurs paramètres de contrôle et en présence d'événements accidentels. Les figures 14 et 15 montrent la variation de l'indice de fiabilité $\beta$. en fonction de deux paramètres de dimensionnement : la hauteur de recouvrement $\mathrm{H}$ et la largeur de la tranchée B dans le cas d'une conduite D2000 BA en présence d'un mauvais bourrage au niveau des flans du tuyau. Ces figures présentent les courbes d'isovaleurs et les représentations en surface de $\beta$, en fonction de $H / D$ et $B / D$, $D$ étant le diamétre de la conduite :

Ce type de visualisation présente l'intérêt de mettre en évidence les paramètres prépondérants et permet de choisir des combinaisons de paramètres de robustesse conduisant à une fiabilité maximale. Elle nous permet aussi, pour un niveau de fiabilité donné, de fixer les valeurs seuil des paramètres de contrôle.

\section{5}

\section{Conclusion}

Cet article présente une révision du comportement des conduites enterrées et propose une approche fiabiliste pour l'évaluation de leur sécurité.

En se basant dans un premier temps sur les résultats de différentes études pathologiques, nous avons pu établir l'état actuel des réseaux d'assainissement et en tirer des informations sur les principales caractéristiques du patrimoine français. Il apparait ainsi que $8 \%$ du linéaire total, soit $8.000 \mathrm{~km}$ de canalisations non visitables et $4300 \mathrm{~km}$ de canalisations visitables, nécessitent une réhabilitation. Ceci montre l'ampleur des dégradations, Les conséquences, tant économiques qu'écologiques, se font de plus en plus sentir.

Sur l'ensemble des défauts constatés, il ressort de ces mêmes études que le déboitement et le décalage au niveau des joints sont les défauts les plus fréquents, suivis des fissures, longitudinales ou transversales, et des cassures. Ces résultats ont été confirmés par l'étude que nous avons menée sur un linéaire d'environ $100 \mathrm{~km}$ du réseau d'assainissement de la communauté urbaine du Grand Nancy. En effet, les problèmes de déboitement et de décalage représentent $30 \%$ du pourcentage total des défauts. Les fissures représentent $20 \%$ environ. Ces défauts sont souvent dus à des effets de flexion longitudinale, pour les déboitements et les fissures circulaires, et transversale ou effet d'écrasement, pour les fissures longitudinales. Les causes de ces effets sont multiples et de différentes natures; les mouvements du sol sont des plus courants. L'une des conséquences importantes de ce constat est la nécessité de la prise en compte du comportement axial des conduites enterrées, souvent négligé par les règlements de dimensionnement.

Les données pathologiques étant peu nombreuses et souvent incomplètes, nous avons entrepris la modélisation du système sol-concluite afin de confirmer et de compléter les résultats des études précédentes, d'une part, et de répondre à la démarche de l'approche fiabiliste, d'autre part. Nous avons pris en compte aussi bien le comportement transversal que le comportement axial de la conduite. 


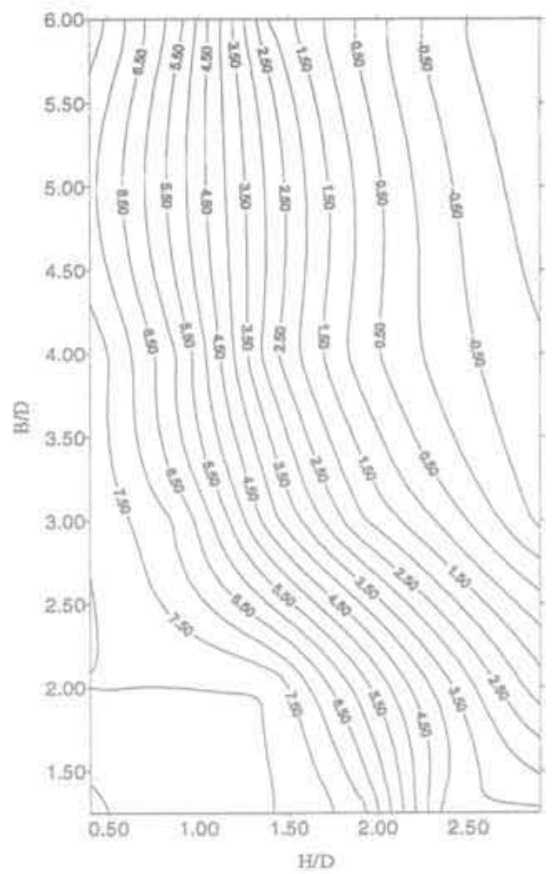

(a) Courbes d'Isovaleurs

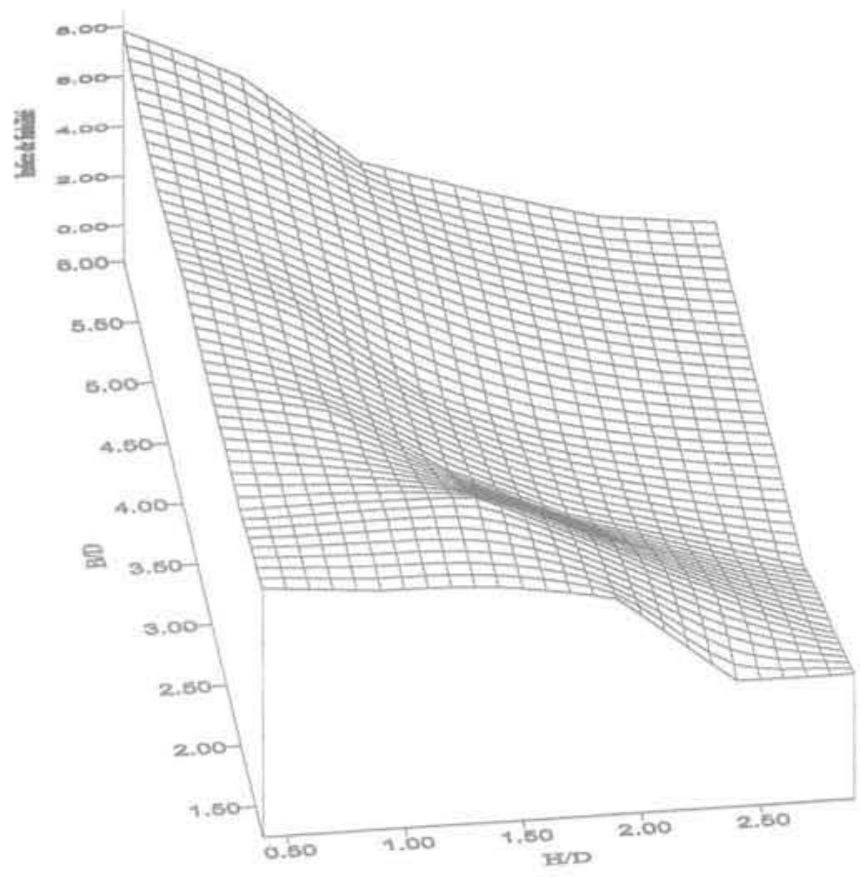

(b) Représentation en surface

FG. 14 Variation de l'indice de fiabilité $\beta_{1}$. Cas du D2000 BA sans surcharges.

Variation of reliability index $\beta$. Case of D2000 BA without surface loading.

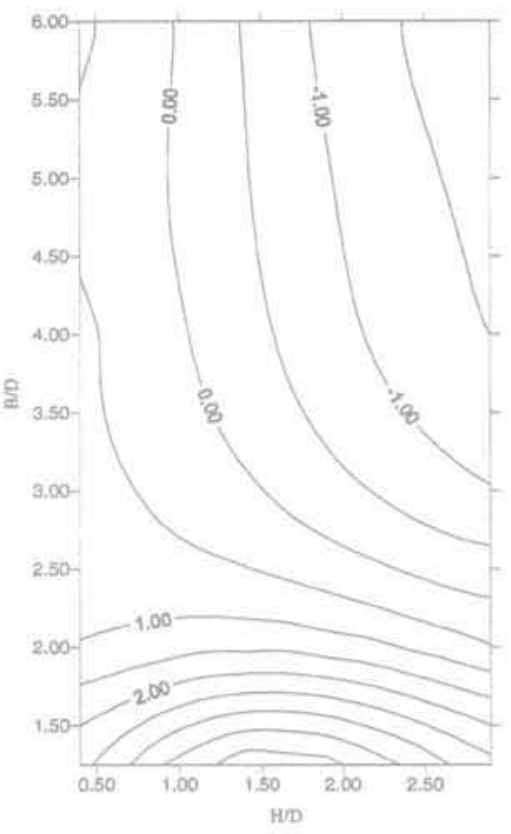

(a) Courbes d'Isovaleurs

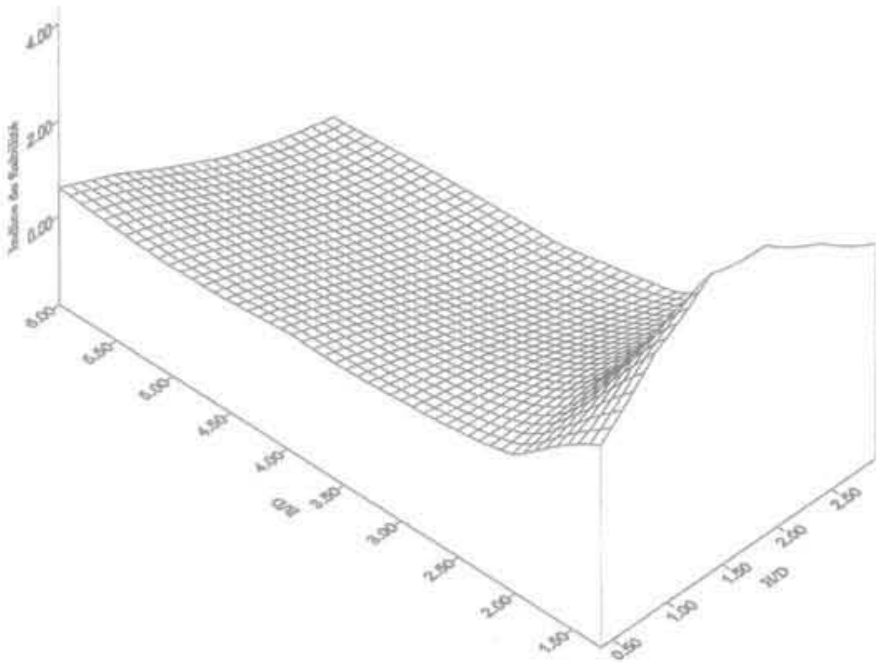

(b) Représentation en surface

FIG. 15 Variation de l'indice de fiabilité $\beta$, . Cas du D2000 BA avec surcharges. Variation of reliability index $\beta$. Case of D2000 BA with surface loading.

Dans la dernière partie de cette étude, nous avons entamé l'analyse de fiabilité. Nous avons défini le système sol-conduite avec ses variables déterministes et aléatoires. Les fonctions de performance ont été définies en prenant en compte les défauts les plus courants. Ainsi, nous avons formulé deux fonctions de perfor- ment transversal $G_{1}$, et de la flexion simple en comportement axial $G_{2}$. Ces deux fonctions de performance nous ont permis d'estimer deux indices de fiabilité. Nous avons choisi d'étudier la sensibilité de ces indices en fonction des différents paramètres du système que nous avons classé dans trois catégories : paramètres de contrôle dont les valeurs et les variations peuvent être 
facilement contrôlées et qui peuvent constituer des̊ facteurs de robustesse, telles que les dimensions de la conduite ou de la tranchée, paramètres accidentels sur lesquels nous n'avons aucune emprise, tels que les vides sous la conduite, les points durs ou les discontinuités du sol, et paramètres intermédiaires qui peuvent constituer des paramètres de contrôle en prenant les mesures nécessaires pour en assurer le suivi, tels que la qualité de bourrage au niveau des hanches de la conduite ou le compactage du remblai de la tranchée, et qui dans le cas contraire deviennent des paramètres accidentels.

Nous avons trouvé que la sensibilité de la fiabilité, exprimée par les deux indices $\beta_{1}$ et $\beta_{2}$, varie de façon nette d'un paramètre à un autre. Les paramètres accidentels sont les plus influents. Les paramètres de contrôle et les paramètres intermédiaires ont, dans cer- tains cas, une aussi grande influence sur la fiabilité que les paramètres accidentels, mais restent en général moins influents. Ainsi, les dimensions de la tranchée (profondeur de pose et largeur de tranchée), notamment pour les grands diamètres, font varier de manière significative l'indice de fiabilité $\beta$. Les caractéristiques de la conduite, quant à elles (élancement L/D ...), agissent moins sur les variations des indices de fiabilité mais leur influence reste certaine. Nous avons montré à ce propos, qu'en cas d'événements accidentels (mauvais bourrage, passage de la conduite d'une zone non compressible à une zone très compressible), les paramètres de contrôle tels que, la profondeur de pose ou l'élancement L/D, peuvent, s'ils sont choisis judicieusement, améliorer la fiabilité de la conduite et, de ce fait, ètre considérés comme des facteurs de robustesse du système.

\section{Bibliographie}

Aflak A. - Elaboration d'un cadre méthodologique pour l'aide à la décision en matière de gestion de la maintenance du réseau technique urbain d'assainissement, thèse de docteur-ingénieur. INSA Lyon, 1994, $300 \mathrm{p}$.

Benjamin J.R., Cornell C.A. - Probability, Statistics and Decisions for Civil Engineers, New York McGraw Hill, 1970. $685 \mathrm{p}$.

Benmansour A. - Fiabilité des conduites enterrées, thèse de doctorat de l'INPL. Ecole nationale supérieure de géologie de Nancy, 1996.

Benmansour A., Abdallah A. Masrouri F. Auvinet G. - Analyse fiabiliste du comportement axial des conduites d'assainissement, Revue Canadienne de Géotechrique (à paraitre)

Benmansour A. Pilloy J.-C. - Etude pathologique de $90 \mathrm{~km}$ du réseau d'assainissement nancéien. Nouveaux constats et leurs prise en compte, $76^{*}$ congrès de I'AGHTM, Londres, 28-31 mai 1996. pp. 523-538

Benmansour A. Auvinet G. Soubra A.H.Validation de lois de comportement des remblais de tranchée dans l'étude des conduites enterrées, $2^{*}$ réunion annuelle GEO 95, 11-15 décembre, 1995, Aussois. 3 p.

Degas G. - Natures et causes des désordres dans les réseaux d'assainissement. Centre d'études et de recherches de l'industie du béton manufacturé (CERIB), Éperon n 84, 1986, 13p.

Duncan J.M. - Behaviour and design of Long-Span metal culvert, Journal of the Geotechnical Engineering Division, 1979. vol. 105, n ${ }^{\circ}$ GT3, pp. 399-418.

Duncan J.M. Drawsky R.H. - Desigh procedures for flexible metal culverts structures. Geotechnical engineering research report UCB/GT/83-02. University of California, Berkeley, 1983.

Duncan J.M., Chang C.Y. - Non-linear analysis of stress and strain in soils, Journal of Soils Mechanics and Foundations Engineering Division, ASCE, 1970. vol. $96, n^{\circ}$ SM5, pp, 1629-1653.

Fages R., Bouyat C. - Modèle mathéma- tique intégrant le comportement irrèversible du sol en état élasto-plastique. Exemple d'application. Étude de l'influence des paramètres, Travaux, $\mathrm{n}^{\circ}$ 441. décembre 1971, pp. 38-46.

Fernando N.S.M., Small J.C., Carter J.P. Elastic analysis of buried structures subject of three-dimensional surface loading, International Journal for Numerical Methods in Geomechanics, 1996. vol. 20, pp. 331-349.

Goulter 1.C. Kazemi A. - Analysis of water distribution pipe failure types in Winnipeg. Canada, Journal of Transportation Engineering, ASCE, 1989, vol. 115. pp.95-111.

Jeyapalan J.K. Abdel-Magid A.M. - Longitudinal stresses and strains in design of RPM pipes, Journal of Transportation Engineering. ASCE, 1987, yol. 113, pp. 315-331.

Jeyapalan J.K., Bolden B.A. - Performance and selection of rigid and flexible pipes. Journal of Transportation Engineering, ASCE, 1986, vol. 112, n 5, pp. 507-524.

Krizek R.J., McQuade P.V. - Behaviour of buried concrete pipe, Journal of the Geotechnical Engineering Division, 1978, vol. 99, n ${ }^{\circ}$ GT7, pp. 815-836.

Le Barbanchon G., Besanval G. Recherche des désordres dans les réseaux d'assainissement des communes rurales en vue de leur réhabilitation. Génje rural, février 1982.

Lester J., Farrar D.M - An examination of the defects observed in six kilometers of sewers, Transport and Road Research Laboratory, Supplementary report SR, Crowthorne, 1979, $28 \mathrm{p}$.

Marston A. - Recent research relative to culvert pipe, Public Roads,1927, vol. 7. pp. 226-229.

Nath P. - Finite element analysis of a large diameter burjed steel pipeline. Transport and Road Research Laboratory (TRRL), Report 778, Crowthorne, Berkshire. England, 1977, $20 \mathrm{p}$.

Nayak G.C., Prakash S., Gupta R. - Finite element analysis of ditch conduits. International Symposium on Soil Structure Interaction, Roorkee, India, 1977, pp. 51-59.
Schwarze C. - Techniques d'auscultation de l'environnement proche des canalisations d'assainissement, thése de docteur-ingénieur, Ecole nationale des ponts et chaussées, Paris, 1994, 150 p.

Seed R. B. Raines J R. - Failure of flexible long-span culverts under exceptional live loads. Transportation Research Record 1191, 1988, pp. 22-29.

Selig E.T., Packard D.L - Buried concrete pipe trench installation analysis, Journal of Transportation Engineering, 1987 . vol. $113, n^{\circ}$ 5, pp. 485-501.

Selig E.T., McVay M.C., Chang C.S. - Finite element modeling of buried concrete pipe installations, Soil-Structure Interaction of Sub-Surface Conduits, Transport Research Record 878, 1982, pp. 17-23,

Selvadurai A.P.S. - Mechanics of buried pipelines induced by random ground movements, proceedings of the Engineering Mechanics Symposium, Vancouver, 1991, pp. 142-151.

Selvadurai A.P. S - Numerical simulation of soil pipe interaction in a ground subsidence zone, proceedings of the International Conference in Advances in Underground Pipeline Engineering, Madison, 1985, pp. 311-319.

Selvadurai A.P.S., Pang S. - Nonlinear effects in soil pipe interaction in a ground subsidence zone, proceedings of the 6 th International Conference on Numerical Methods in Geomechanics, Innsbruck. Austria, 1988, pp. 1085-1094

Selvadurai A.P.S.. Au M.C., Shinde S.B. Solt-pipe interaction in a pipeline with prescribed displacements, proceedings of the first National Conference on Flexible Pipes, Columbus, Ohio. A. A Balkema, Rotterdam, 1990

Spangler M.G. - The structural design of flexible pipe culverts, Iowa State College Bulletin, Ames, $n^{\circ} 153,1941,84$ p.

Valliappan S., Matsuzaki K., Raja Sekar H.L. - Nonlinear stress analysis of buried pipes, Uniciv Report n R164. November. University of new south wales, Kensington, Australia, 1976, $17 \mathrm{p}$. 